\title{
Prostate and Bladder Metastases of Malignant Melanoma: Case Report
}

\author{
(D) Ali Gürağaç MD', (1) Sercan Yılmaz MD², (1) Zafer Demirer MD³, (1) Hasan Cem Irkılata MD4, (D) Yusuf Kibar MD \\ 'Bitlis State Hospital, Clinic of Urology, Bitlis, Turkey \\ 2University of Health Sciences, Gülhane Research and Training and Research Hospital, Clinic of Urology, Ankara, Turkey \\ ${ }^{3}$ Eskișehir Yunus Emre State Hospital, Clinic of Urology, Eskișehir, Turkey \\ ${ }^{4}$ Private Davraz Life Hospital, Clinic of Urology, Isparta, Turkey \\ ${ }^{5}$ Private Koru Hospital, Clinic of Urology, Ankara, Turkey
}

\begin{abstract}
Prostate or bladder metastases of malignant melanoma (MM) are rarely encountered in clinical practice. To our knowledge, coexistence of prostate and bladder metastasis of MM has not been reported to date. We present the case of a 58-year-old male who was diagnosed with MM of the umbilical skin 2.5 years earlier and who presented to us with intermittent painless macroscopic hematuria and lower urinary tract symptoms. The prostate and bladder lesions were removed as completely as possible by transurethral resection. Pathological examination demonstrated simultaneous prostate and bladder metastases of MM.
\end{abstract}

Keywords: Bladder, malignant melanoma, metastases, prostate

\section{Introduction}

Secondary tumors of prostate and bladder are usually seen as metastasis or direct tumoral extension (1). Metastasis of malignant melanoma (MM) to prostate or bladder rarely causes hematuria and lower urinary tract symptoms (LUTS) before it becomes a systemically manifest disease (1). Although, prostate or bladder metastasis of MM generally detected during autopsy and extremely rare in clinical practice, with less than 20 cases reported for bladder metastasis and only 2 cases reported for prostate metastasis of $\mathrm{MM}$ in the last 30 years in the English literature (2). We report a case of simultaneously detected MM metastases into the prostate and bladder. To our knowledge, this is the first reported case of metastatic MM of the prostate and bladder identified simultaneously.

\section{Case Report}

A 58-year-old male patient presented with recurrent painless gross hematuria for one month and LUTS for 3 months. He had a history of wide local excision for his umbilical mass and inguinal lymph node dissection 2.5 years ago, which is diagnosed as MM pathologically. He had received interferon alpha treatment for 2 years until bilateral pulmonary metastasis and mediastinal lymph adenopathy occurred 4 months ago. Physical examination revealed excision scar on the umbilicus and right lower quadrant. Digital rectal examination revealed an asymmetrically enlarged, irregular and firm prostate. Prostatespecific antigen level was within normal limits $(0.62 \mathrm{ng} / \mathrm{mL})$. Urinalysis, urine culture and urine cytology were normal except hematuria. Ultrasonographic evaluation revealed moderate hydronephrosis on the right kidney and increased bladder wall thickness, especially, at the right side with irregular borders, hypoechoic segmental lesions occupied almost whole prostate and bladder are also demonstrated. Magnetic resonance imaging of the abdomen showed filling defect and wall thickening at the right side of bladder and multiple lymph adenopathy located on obturator, para-iliac and inguinal regions. Large mass lesion in the bladder with prostatic involvement and right ureterohydronephrosis also noted.

During cystoscopy, darkly (black) pigmented lesions were seen on the prostatic urethra, bladder neck and trigone (Figure $1 \mathrm{a}, \mathrm{b}$ and $\mathrm{c})$. Then, palliative transurethral resection of the 
lesions was performed (Figure 1a and 1c). After transurethral resection of lesions, hematuria ceased and the LUTS subsided. Histologic sections obtained from both prostate and bladder neck composed of sheets of the neoplastic cells with large eosinophilic cytoplasm and large and darkly basophilic nuclei (Figure 2a and 2b).During cystoscopy, darkly (black) pigmented lesions were seen on the prostatic urethra, bladder neck and trigone (Figure 1a, b, c). Then, palliative transurethral resection of the lesions was performed (Figure 1a, c). After transurethral resection of lesions, hematuria ceased and the LUTS subsided. Histologic sections obtained from both prostate and bladder neck composed of sheets of the neoplastic cells with large eosinophilic cytoplasm and large and darkly basophilic nuclei (Figure 2a, b). Immunohistochemical (IHC) study illustrated human melanoma black-45 and MART1 positivity which are rather specific for MM (Figure 2c, d). Some of these cells also had conspicuous nucleoli and some of them contained intracytoplasmic melanin pigmentation (Figure 2b, e). Informed consent form was obtained from the patient.

\section{Discussion}

$\mathrm{MM}$ is an aggressive neoplasm and its incidence is increasing worldwide (3). MM is potentially curable when detected early and treated appropriately. Recurrence and metastasis are not uncommon, which also carries a grave prognosis with significant morbidity and mortality (4). Except the regional

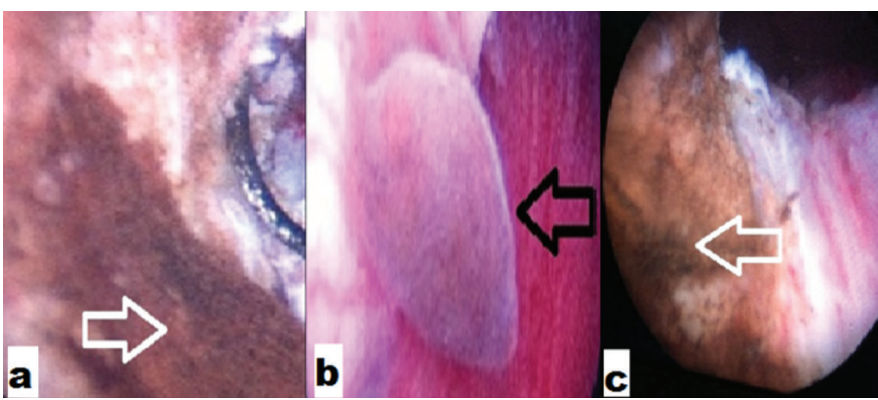

Figure 1. a, b, and c) Transurethral resection of lesions which located at bladder and prostate performed at the same time to relief his symptoms

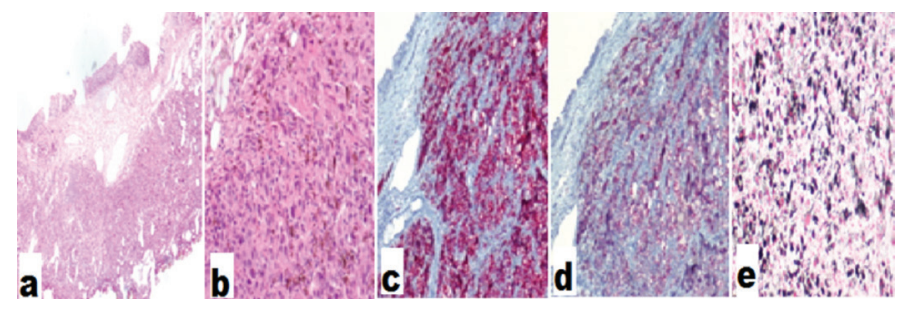

Figure 2. a) Neoplastic infiltration beneath the urothelium (hematoxylin and eosin, 20x). b) The same tumor is also seen in the prostate. Tumor cells contained an intracytoplasmic brown pigment (melanin) (hematoxylin and eosin, 100x). c) Human melanoma black-45 and d) MART1 immunoreactivity (100x immunohistochemical). e) Histochemical staining with Masson Fontana verified the existence of intracytoplasmic melanin pigment (Masson Fontana, 200x) lymph nodes, most common metastasis sites for MM are lungs, liver and brain. Hence, its metastasis can be seen any organs of the human body, clinicians can be faced with various clinical presentation related with affected organs $(1,3)$.

Involvement of urinary system and male genital organs by a secondary tumor can be seen either as a direct tumoral extension or metastasis (1). Metastatic neoplasm of the urinary bladder and prostate are extremely rare and $2 \%$ of the bladder tumors and $2.1 \%$ of the prostate tumors are related with metastases from the other primary tumors, respectively $(5,6)$. Actually, genitourinary system is a common site for metastasis of $\mathrm{MM}$, as many as $37 \%$ metastases had been found in autopsy series of patients with MM. Probably other organ metastasis might have caused dead of the patients with MM before the emergence of urinary symptoms (7).

Most common presenting complaints of urinary metastasis of MM are LUTS and painless macroscopic hematuria (2). Although LUTS might be more prominent in patients with prostatic metastasis than bladder metastasis, symptomatic ambiguity and resemblance with benign prostatic hyperplasia, overactive bladder or urinary tract infection symptoms may lead to misdiagnosis and delay correct therapy $(2,5)$. Unfortunately, macroscopic hematuria is a delayed clinical sign of locally advanced disease for bladder metastasis of $\mathrm{MM}(2,4)$.

Though urine cytology may have detected melanoma cells in prostate and bladder metastases of MM, cytomorphology and special stain must be combined with previous clinical history of patient's melanoma for the accurate diagnosis cystoscopic findings (8). Clinicians must be remember that, without site predominating, the location of the primary lesion is also highly variable and metastatic lesions of the $\mathrm{MM}$ could be detected soon after or over 20 years later (2).

Systemic metastasis of MM are compatible with stage 4 disease and these patients have poor median survival of 6-10 months and less than $5 \%$ surviving more than 5 years $(8,9)$. First sites of the metastasis, number of the metastases and resectability of the metastasis are very important prognostic factors about survival rates (4). Non-visceral metastases at first relapse i.e. in skin, subcutaneous tissues, distant lymph nodes and lung also related with better survival rate than visceral metastasis i.e. in liver, bone and brain (4).

Treatment approaches in the urinary metastasis consists of conservative management that includes radiotherapy, chemotherapy, immunotherapy, and endoscopic resection of the lesions for moderating symptoms. Partial or complete cystectomy and radical prostatectomy should be thought as a treatment modality for the patient potentially curable with solitary metastasis and with longer life expectancy $(4,8,9)$.

Despite extensive clinical researches and promising development about immune modulation and numerous immunotherapy strategies, the treatment options for metastatic MM have been limited, therefore the prognosis of this entity is still poor regardless of treatment modality and the survival rate of these patients has not changed over the past 30 years $(4,10)$. Management of metastatic MM should be tailored to the patient's condition, symptoms and number and size of metastases, clinicians should be considering a poor prognosis of stage 4 disease when choosing treatment modality also. 


\section{Ethics}

Informed Consent: Informed consent form was obtained from the patient.

Peer-review: Externally peer-reviewed.

\section{Authorship Contributions}

Concept: H.C.I., Design: A.G., Z.D., Data Collection or Processing: A.G., S.Y., Analysis or Interpretation: H.C.I., Y.K., Literature Search: S.Y., Writing: A.G., Z.D.

Conflict of Interest: No conflict of interest was declared by the authors.

Financial Disclosure: The authors declared that this study received no financial support.

\section{References}

1. Morichetti D, Mazzucchelli R, Lopez-Beltran A, et al. Secondary neoplasms of the urinary system and male genital organs. BJU Int 2009;104:770-776.

2. Paterson A, Sut M, Kaul A, et al. Metastatic malignant melanoma of the urinary bladder: Case report and literature review. Cent European J Urol 2012;65:232-234.
3. Jemal A, Siegel R, Xu J, et al. Cancer statistics, 2010. CA Cancer J Clin 2010;60:277-300.

4. Barth A, Wanek LA, Morton DL. Prognostic factors in 1, 521 melanoma patients with distant metastases. J Am Coll Surg 1995;181:193-201.

5. Bates AW, Baithun SI. Secondary neoplasms of the bladder are histological mimics of nontransitional cell primary tumours: clinicopathological and histological features of 282 cases. Histopathology 2000;36:32-40.

6. Bates AW, Baithun SI. Secondary solid neoplasms of the prostate: a clinico-pathological series of 51 cases. Virchows Arch 2002;440:392-396.

7. de la Monte SM, Moore GW, Hutchins GM. Patterned distribution of metastases from malignant melanoma in humans. Cancer Res 1983;43:3427-3433.

8. Martinez-Giron R. Melanoma cells in voided urine cytology. Cytopathology 2008;19:402-403.

9. Balch CM, Gershenwald JE, Soong SJ, et al. Final Version of 2009 AJCC Melanoma Staging and Classification. J Clin Oncol 2009;27:6199-6206.

10. Batus M, Waheed S, Ruby $C$, et al. Optimal management of metastatic melanoma: current strategies and future directions. Am J Clin Dermatol 2013;14:179-194. 